\title{
Impiego di tramadolo nelle diverse patologie dolorose
}

\author{
Orietta Zaniolo*
}

\begin{abstract}
Pain represents a major health problem that afflicts a significant number of patients, resulting in personal suffering, reduced productivity and substantial health care costs, specially in chronic conditions. Chronic pain, such as low back pain, osteoarthritis, neuropathic and oncologic pain, requires a global management based on clinical evaluation, defining of the adequate therapeutic strategy and assiduous cares.

Generally, patients with chronic pain need two different analgesic formulation: one at fixed doses and prolonged duration of action for control of baseline pain and one fast-release drug for treatment of breakthrough pain. Main guidelines recommend use of the same active principle for both these formulations. Tramadol, a dual action analgesic agent, used for controlling moderate to severe pain control in acute and chronic diseases, is available in several different oral formulations. This is important because, apart from efficacy and tolerability, that are the most important criteria to evaluate a therapy, the choice of a formulation with an uncomplicated dosing regimen can increase compliance and, consequently, clinical results, and ameliorate patient quality of life.

For the treatment of chronic pain, the appropriate analgesic dosage can be achieved through a gradual titration: this permits to minimize adverse effects and cost of therapy. With tramadol this action can be realized with $50 \mathrm{mg}$ extended-release capsules, that represent the minimal effective dose.

When adequate pain relief is obtained, it's possible to reduce frequency and complexity of dosing regimen using a new once-daily tablet of tramadol.

Fast-release orodispersible tramadol tablet, with its practical advantage (rapid disintegration in the mouth without need of water), can be used for the control of breakthrough and acute pain.

The cost of treatment with tramadol results relatively low, also when two daily episodes of breakthrough pain are considered.
\end{abstract}

Keywords: tramadol, chronic pain, cost of treatment, compliance

Farmeconomia e percorsi terapeutici 2005; 6 (4): 385-396

\section{INTRODUZIONE}

L'Associazione Internazionale per lo Studio del Dolore (IASP) definisce il dolore come "Una sgradevole esperienza sensoriale ed emotiva, associata ad un effettivo o potenziale danno tissutale o comunque descritta come tale. Il dolore è sempre un'esperienza soggettiva. Ogni individuo apprende il significato di tale parola attraverso le esperienze correlate ad una lesione durante i primi anni di vita. Sicuramente si accompagna ad una componente somatica, ma ha anche carattere spiacevole, e perciò, ad una carica emozionale" [1].

Il dolore, in generale, può essere di tipo acuto o cronico. Il dolore acuto è finalizzato ad allertare il corpo sulla presenza di stimoli pericolosi o potenzialmente tali nell'ambiente o nell'organismo stesso. È, quindi, un dolore che può essere definito "utile", che prima di essere trattato va interpretato, in modo da agire, quando è possibile, direttamente sulla patologia causale.

Il dolore cronico non rappresenta solo un'estensione temporale del dolore acuto, ma assume caratteristiche qualitative completamente diverse, che necessitano di un approccio mentale, culturale e professionale più approfondito.

In particolare, il dolore cronico presente nelle malattie degenerative, neurologiche e oncologiche, specie nelle fasi avanzate e terminali di malattia, assume caratteristiche di dolore globale, con conseguenze che intaccano la sfera fisica, psicologica e sociale [2].

Una gestione globale del dolore cronico comprende la valutazione del dolore, l'impostazione di una strategia terapeutica appropriata e l'assistenza continua. A causa del- 
la grande variabilità delle patologie associate a stimoli dolorosi, delle caratteristiche peculiari del paziente e della frequente presenza di comorbidità la strategia terapeutica deve essere fortemente individualizzata. Oltre a efficacia e tollerabilità, che devono essere $\mathrm{i}$ primi criteri in base ai quali impostare la terapia, la scelta di formulazioni altamente maneggevoli e l'impostazione di schemi posologici in grado di incontrare il più possibile le esigenze del paziente possono aumentare l'efficienza della prescrizione e migliorare la qualità di vita.

Scopo del presente lavoro è quello di esaminare l'impiego di tramadolo nelle diverse situazioni algiche, analizzando vantaggi e svantaggi, clinici ed economici, legati all'uso delle diverse formulazioni in cui è disponibile questo farmaco.

Tramadolo, analgesico oppioide indicato per il trattamento del dolore da moderato a grave, è disponibile in commercio in diverse forme farmaceutiche, alcune delle quali (compresse once-daily e orodispersibili) introdotte recentemente in commercio al fine di aumentare la sua maneggevolezza e permettere il più possibile la personalizzazione dello schema posologico.

Tramadolo per la somministrazione orale, sul quale focalizzeremo la nostra attenzione, è disponibile in gocce $100 \mathrm{mg} / \mathrm{ml}$, in compresse orodispersibili da $50 \mathrm{mg}$, in capsule e in compresse effervescenti da $50 \mathrm{mg}$ per quanto riguarda le formulazioni a rilascio immediato (IR), in compresse da 100, 150 e $200 \mathrm{mg}$ e capsule da $50,100,150$ e $200 \mathrm{mg}$, somministrabili 2 volte al giorno, e in compresse once-daily (OD) da 150 e $200 \mathrm{mg}$, per quelle a rilascio prolungato (SR).

Studi farmacocinetici hanno indagato e confrontato i parametri di assorbimento, distribuzione e eliminazione delle varie formulazioni [3-7].

Tramadolo in formulazione orale a rilascio immediato (IR) raggiunge la concentrazione plasmatica massima entro 2 ore dalla somministrazione e presenta un'emivita di eliminazione di circa 5-6 ore, richiedendo, per la copertura delle 24 ore, una frequenza di 4-6 somministrazioni giornaliere.

Tramadolo SR a rilascio controllato presenta un assorbimento prolungato e un'emivita apparente notevolmente allungata che permette la semplificazione dello schema posologico fino a due somministrazioni al giorno. Inoltre, l'assenza di improvvisi picchi plasmatici e di repentine diminuzioni di concentrazione comportano una migliore tollerabilità e una diminuzione della ricomparsa del dolore intermittente (fenomeno picco-valle) rispetto alle formulazioni IR $[5,8]$. Tramadolo a rilascio controllato è disponibile in compresse SR (unità singole a matrice unica) e in capsule SR (dosi multiple costituite da microgranuli). Le due formulazioni sono equivalenti per quanto riguarda la quantità di tramadolo assorbito (AUC), ma le capsule producono un picco plasmatico (Cmax) inferiore e più ritardato e un emivalore maggiore rispetto alle compresse. In un recente studio di confronto, la variabilità inter- e intra-individuale della biodisponibilità è risultata significativamente inferiore per la capsula rispetto alla compressa $(p=0,04)$. Gli autori dello studio hanno imputato tale risultato al fatto che, mentre i granuli di cui è composta la capsula, avendo un diametro inferiore a $2 \mathrm{~mm}$, passano nel piccolo intestino indipendentemente dal grado di svuotamento gastrico, il passaggio di una compressa lungo il tratto gastrointestinale dipende dal ritmo di apertura del piloro, il quale è influenzato da diversi fattori (assunzione cibo, esercizio fisico, umore, postura, condizioni patologiche, farmaci e stress) [5].

La realizzazione di compresse costituite da un tipo di matrice lipofila (idrofobica) permette, grazie alle sue caratteristiche chimico-fisiche, di rallentare il rilascio del principio attivo in misura maggiore rispetto alla matrice idrofila delle formulazioni SR in modo da rendere possibile l'utilizzo di tramadolo in somministrazione unica giornaliera (OD); la curva rilascio/tempo relativa alla matrice lipofila mostra, infatti, come solo dopo circa 20 ore il farmaco è completamente dissolto [9]. In seguito a questa cinetica modificata, l'emivalore risulta di circa 16 ore [10]. Questo tipo di compressa determina una disponibilità sistemica del farmaco, dopo correzione per la dose, simile a quella riscontrabile dopo assunzione di tramadolo IR. Uno studio condotto da Tagarro e colleghi ha confrontato il profilo farmacocinetico delle compresse orodispersibili di tramadolo con quello delle capsule IR standard. L'area sotto la curva concentrazione/tempo, la massima concentrazione plasmatica, il tempo in cui essa viene raggiunta e il valore di T1/2 sono risultati molto simili dopo assunzione delle due formulazioni [4]. Poiché indagini conoscitive indicano che, 40 minuti dopo 1'assunzione del farmaco orodispersibile, i valori plasmatici di tramadolo e del suo metabolita risultano maggiori di quelli osservabili dopo somministrazione delle capsule standard, gli autori dello studio hanno avanzato l'ipotesi che il maggior livello plasmatico nelle fasi iniziali fosse riconducibile a un parziale assorbimento pregastrico del tramadolo orodispersibile attraverso la mucosa orale [4].

Requisiti fondamentali affinché l'obiettivo terapeutico sia raggiunto sono l'efficacia e la tollerabilità del trattamento farmacologico; tuttavia per massimizzare l'efficienza terapeutica è importante che l'aderenza alla terapia sia completa. La relazione fra compliance e frequenza di somministrazione è stata studiata in diversi 
trial; in particolare una review pubblicata nel 2003 ha dimostrato che la riduzione del numero di somministrazioni/die, attraverso formulazioni a lento rilascio o introduzione di nuovi principi attivi, migliora l'aderenza alla terapia, la soddisfazione del paziente e i costi [11]. Da un'altra review è emerso che la percentuale di farmaco prescritto realmente assunta passa, in media, dal $79 \%$ per i farmaci somministrati una volta al giorno, al $51 \%$ per i farmaci somministrati quattro volte/die [12].

Il vantaggio di uno schema posologico semplice diventa più evidente in caso di pazienti che necessitano di terapie protratte nel tempo, evitando dimenticanze di assunzione del farmaco e confusione fra medicinali diversi, in particolare per i pazienti anziani ed in politerapia. Anche la maneggevolezza della formulazione farmaceutica può contribuire ad aumentare l'aderenza del paziente alla terapia: modalità di somministrazione semplici e sicure possono, infatti, facilitare il completo rispetto della prescrizione.

Le formulazioni di tramadolo recentemente introdotte sono state ideate proprio al fine di aumentare la compliance: le compresse OD semplificano lo schema posologico, riducendo l'impatto della terapia sulle abitudini e sullo stile di vita del paziente, mentre le compresse orodispersibili possono rappresentare un'opzione terapeutica di facile utilizzo in quanto, dissolvendosi già nella cavità orale, possono essere assunte in ogni momento, senza la necessità di accompagnarle con liquidi e anche nei casi in cui il paziente ha problemi di deglutizione o, semplicemente, a inghiottire le compresse tradizionali. Altre formulazioni di tramadolo IR, come la formulazione in gocce, possono risultare meno sicure dal punto di vista del dosaggio: il conteggio del numero di gocce, atto per molti banale, per alcuni pazienti, in particolare quelli anziani, può essere motivo di preoccupazione e di errori, che conducono all'assunzione di dosi imprecise. In tali casi, per il medico curante, risulta più difficile capire se l'eventuale comparsa di effetti collaterali si è verificata a dosaggi terapeutici o no, oppure valutare se la persistenza del dolore sia dovuta a inefficacia del farmaco o all'assunzione non corretta della dose prescritta.

La comparsa di eventi avversi rappresenta una delle principali cause di abbandono della terapia o di compliance parziale. Gli effetti collaterali più frequentemente associati a tramadolo sono nausea, vomito e vertigini; essi, pur essendo transitori e solitamente di entità da lieve a moderata, inducono l'interruzione del trattamento nel $20 \%$ dei casi circa $[3,13]$.

Per ridurre o prevenire la loro comparsa, è utile iniziare la terapia a basso dosaggio e aumentarlo gradatamente, assumere il farmaco la sera prima di coricarsi e, in caso di precedente comparsa di tali effetti, associare un antiemeti- co durante i primi giorni di trattamento. In molti casi proseguendo la terapia, per un fenomeno di adattamento recettoriale, tali effetti diminuiscono di frequenza/intensità (fenomeno di tolleranza a tali eventi avversi) [14].

\section{IMPIEGO DI TRAMADOLO NELLE DIVERSE PATOLOGIE DOLOROSE}

\section{Dolore cronico}

Il sintomo "dolore" è altamente variabile e soggettivo, anche all'interno di un gruppo di pazienti affetti dalla stessa patologia. La minima dose di antidolorifico in grado di controllare in modo soddisfacente il dolore dipende dall'intensità dello stesso e dalla risposta clinica del singolo paziente; essa viene ricercata individualmente mediante titolazione graduale del farmaco, per minimizzare gli effetti collaterali ed evitare un inutile spreco di risorse.

Una volta raggiunta la dose efficace, le principali linee guida internazionali raccomandano che la gestione del dolore cronico avvenga mediante l'utilizzo di due diverse formulazioni dello stesso farmaco: una a lunga durata d'azione, assunta ad orari fissi per la copertura antalgica di base e una a rilascio immediato per eventuali episodi di riacutizzazione del dolore (breakthroug pain) che possono insorgere improvvisamente e in assenza di fattori scatenanti. Solitamente la comparsa di questi picchi di dolore ha frequenza variabile (da 1 a 6 al giorno) e durata di circa 20-30 $\min [15,16]$.

La disponibilità di tramadolo in numerose formulazioni permette di gestire le diverse fasi del trattamento, e le varie esigenze terapeutiche, mediante l'uso dello stesso principio attivo e di schemi posologici semplici.

Le capsule a rilascio controllato da $50 \mathrm{mg}$ possono essere utilmente impiegate per la titolazione del farmaco; questa formulazione risulta particolarmente adatta a tal fine in quanto $50 \mathrm{mg}$ è considerata la dose unica standard minima efficace, il rilascio prolungato del farmaco ne permette la somministrazione due volte al giorno e la formulazione ad unità multiple sembra garantire, come detto in precedenza, picchi plasmatici meno repentini e maggior prevedibilità dell'effetto, rispetto alla formulazione SR in compresse.

Alcuni studi hanno dimostrato che, essendo gli effetti collaterali di tramadolo dose-dipendenti, l'aumento graduale del dosaggio, inizialmente molto basso, ne migliora la tollerabilità; un recente studio ha indagato l'effetto di uno schema di titolazione molto semplice: somministrazione iniziale di tramadolo in capsule SR $50 \mathrm{mg}$ due volte al giorno e, dopo circa sette giorni, passaggio al dosaggio di 100 $\mathrm{mg}$ due volte al giorno vs. schema convenzionale (100 mg SR due volte al giorno per tutta la durata del trattamento). 


\begin{tabular}{|c|c|c|c|c|}
\hline & $\begin{array}{c}\text { Forma } \\
\text { farmaceutica }\end{array}$ & $\begin{array}{c}\text { Schema } \\
\text { posologico }\end{array}$ & $\begin{array}{c}\text { Costo giornaliero } \\
\text { (euro) }\end{array}$ & $\begin{array}{c}\text { Costo mensile } \\
\text { (euro) }\end{array}$ \\
\hline \multirow[t]{3}{*}{ Titolazione: } & $\begin{array}{l}\text { Tramadolo } 30 \\
\text { cps SR } 50 \text { mg }\end{array}$ & 2 cps/die & 0,42 & \multirow{3}{*}{ Non rilevante } \\
\hline & & 3 cps/die & 0,63 & \\
\hline & & 4 cps/die & 0,84 & \\
\hline \multirow[t]{2}{*}{ Mantenimento } & Tramadolo cpr OD 150 mg & $1 \mathrm{cpr} / \mathrm{die}$ & 0,63 & 19,06 \\
\hline & Tramadolo cpr OD 200 mg & $1 \mathrm{cpr} / \mathrm{die}$ & 0,84 & 25,40 \\
\hline $\begin{array}{l}\text { Controllo } \\
\text { riacutizzazioni }\end{array}$ & $\begin{array}{l}\text { Tramadolo } 20 \mathrm{cpr} \\
\text { orodispersibili } 50 \mathrm{mg}\end{array}$ & $\begin{array}{c}1 \text { cpr orodispersibile al } \\
\text { bisogno (frequenza } \\
\text { considerata: } 0-2 \text { episodi/die* }{ }^{\star} \text {. }\end{array}$ & $\begin{array}{l}\text { - } 0 \text { (no episodi) } \\
\text { - 0,23 (1 episodio) } \\
\text { - 0,46 ( } 2 \text { episodi) }\end{array}$ & $\begin{array}{c}- \\
6,9 \\
13,8\end{array}$ \\
\hline \multicolumn{5}{|c|}{$\begin{array}{l}\text { Tabella I } \\
\text { Costo giornaliero di tramadolo per il trattamento del dolore cronico nelle diverse fasi terapeutiche. I prezzi considerati sono quelli al } \\
\text { pubblico. I prezzi per il Sistema Sanitario Nazionale sono leggermente inferiori grazie allo sconto (del } 4 \% \text { circa) applicato dalle } \\
\text { farmacie al SSN }\end{array}$} \\
\hline
\end{tabular}

L'efficacia dei due schemi posologici è risultata simile, ma i pazienti che inizialmente avevano assunto $50 \mathrm{mg}$ di farmaco hanno riportato reazioni avverse meno frequentemente. La proporzione di pazienti che ha interrotto la terapia in seguito a comparsa di eventi avversi è stata pari a 5,6\% nel gruppo con aumento della dose e $12,6 \%$ nel gruppo con dose costante [14].

In linea di massima, lo schema di titolazione mediante capsule SR consigliato prevede: inizio della terapia con due capsule SR da $50 \mathrm{mg}$ al giorno, pari a $100 \mathrm{mg} / \mathrm{die}$, per un minimo di 3 giorni, in caso di persistenza del dolore aggiunta di un'altra capsula SR da $50 \mathrm{mg}$, preferibilmente la sera, e ulteriore aumento della posologia nel caso in cui $150 \mathrm{mg} /$ die non siano ancora sufficienti. La dose massima di tramadolo raccomandata è di $400 \mathrm{mg} /$ die [17].

Raggiunta la minima dose in grado di controllare il dolore, è possibile, al fine di semplificare la posologia e facilitare nel lungo periodo l'aderenza alla terapia, passare alla formulazione di tramadolo once-daily; in base al dosaggio giornaliero minimo che ha indotto completa analgesia, verrà somministrata una compressa OD da 150 o da $200 \mathrm{mg}$.

Le formulazioni di tramadolo a rilascio immediato mantengono la loro utilità per il trattamento delle esacerbazioni acute di dolore (breakthrough pain); per la loro maneggevolezza l'utilizzo delle compresse orodisperdibili per il trattamento di questi episodi può rappresentare una valida alternativa alle compresse tradizionali o alle gocce. La frequenza di assunzione può mediamente variare ogni $6-8$ ore; tenendo presente che la somma complessiva delle dosi assunte giornalmente (analgesia di base + rescue medication) non dovrebbe comunque superare i $400 \mathrm{mg} / \mathrm{die}$.

Oltre alla titolazione iniziale, specialmente in patologie progressive come quelle oncolo- giche, la terapia di mantenimento potrebbe, nel tempo, risultare insufficiente a causa del peggioramento del quadro clinico; un utile indicatore dell'aggravamento del dolore è dato dalla frequenza con cui il paziente fa uso di rescue medication: secondo 1'OMS, una frequenza superiore a due episodi di breakthroug pain al giorno è, in molti casi, indicativo di un peggioramento della sintomatologia e quindi della necessità di modificare la terapia di fondo [18].

Riportiamo in Tabella I e II il costo giornaliero del trattamento con tramadolo secondo gli schemi terapeutici finora illustrati.

Come si evince dalle Tabelle I e II, la terapia a base di tramadolo, anche in caso di più episodi di breakthrough pain al giorno, ha costi relativamente bassi.

Riassumiamo di seguito le principali patologie dolorose croniche in cui tramadolo viene utilizzato, sottolineando vantaggi e svantaggi rispetto alle principali alternative terapeutiche.

\section{Dolore muscolo-scheletrico cronico}

Circa 2/3 circa dei casi di dolore cronico sono imputabili ad affezioni muscolo-scheletriche; tra queste l'osteoartrosi e le malattie correlate al lavoro rappresentano le patologie con più alta prevalenza [19-22].

L'osteoartrosi è una malattia degenerativa delle articolazioni dovuta all'erosione delle cartilagini, che può essere idiopatica o secondaria a troppo carico o a una condizione pre-esistente come la sublussazione dell'anca, un'eccessiva deposizione di calcio, un trauma. Questa patologia degenerativa si localizza più frequentemente a livello dell'articolazione dell'anca (coxartrosi), del ginocchio (gonartrosi) o dei dischi vertebrali (spondiloartrosi).

Secondo il National Center for Health Statistic (NCHS) statunitense, nei paesi industrializzati circa il $23-25 \%$ della popolazione di 


\begin{tabular}{|c|c|c|c|c|}
\hline $\begin{array}{l}\text { Dose } \\
\text { giornaliera }\end{array}$ & $\begin{array}{c}\text { Forma } \\
\text { farmaceutica }\end{array}$ & $\begin{array}{l}\text { Schema } \\
\text { posologico }\end{array}$ & $\begin{array}{c}\text { Costo giornaliero } \\
\text { (euro) }\end{array}$ & $\begin{array}{l}\text { Costo mensile } \\
\text { (euro) }\end{array}$ \\
\hline $\begin{array}{l}150 \mathrm{mg}+ \\
\text { rescue } \\
\text { medication }\end{array}$ & $\begin{array}{l}\text { Tramadolo cpr OD } 150 \text { mg + } \\
\text { tramadolo cpr orodispersibili } \\
50 \text { mg }\end{array}$ & $\begin{array}{l}1 \text { cpr OD + } \\
0-1-2 \text { cpr orodispersibili }\end{array}$ & $\begin{array}{l}0,63+ \\
0 \text { (no episodi) - } \\
0,23 \text { ( } 1 \text { episodio) - } \\
0,46 \text { ( } 2 \text { episodi) }\end{array}$ & Range \# 18,9-32,7 \\
\hline $\begin{array}{l}200 \mathrm{mg}+ \\
\text { rescue } \\
\text { medication }\end{array}$ & $\begin{array}{l}\text { Tramadolo cpr OD } 200 \text { mg + } \\
\text { tramadolo cpr } \\
\text { orodispersibili } 50 \text { mg }\end{array}$ & $\begin{array}{l}1 \mathrm{cpr} \text { OD }+ \\
0-1-2 \mathrm{cpr} \text { orodispersibili }\end{array}$ & $\begin{array}{l}0,84+ \\
0 \text { (no episodi) - } \\
0,23 \text { ( } 1 \text { episodio) - } \\
0,46 \text { ( } 2 \text { episodi }\end{array}$ & Range \# 25,2-39 \\
\hline \multirow[t]{2}{*}{$\begin{array}{l}300 \mathrm{mg}+ \\
\text { rescue } \\
\text { medication }\end{array}$} & $\begin{array}{l}\text { Tramadolo cps SR } 150 \mathrm{mg} \mathrm{+} \\
\text { tramadolo cpr orodispersibili } \\
50 \mathrm{mg}\end{array}$ & $\begin{array}{l}2 \text { cps SR/die }+ \\
0-1-2 \text { cpr orodispersibili }\end{array}$ & $\begin{array}{l}1,26+ \\
-0 \text { (no episodi) - } \\
0,23 \text { ( } 1 \text { episodio) - } \\
0,46 \text { ( } 2 \text { episodi) }\end{array}$ & Range \# 37,8-51,6 \\
\hline & $\begin{array}{l}\text { Tramadolo cpr OD } 150 \text { mg + } \\
\text { tramadolo cpr orodispersibili } \\
50 \text { mg }\end{array}$ & $\begin{array}{l}2 \text { cpr OD da assumere insieme + } \\
0-1-2 \text { cpr orodispersibili }\end{array}$ & $\begin{array}{l}1,26+ \\
0 \text { (no episodi) - } \\
0,23 \text { ( } 1 \text { episodio) - } \\
0,46 \text { ( } 2 \text { episodi) }\end{array}$ & Range \# 37,8-51,6 \\
\hline \multirow[t]{2}{*}{$\begin{array}{l}400 \mathrm{mg}+ \\
\text { rescue } \\
\text { medication }\end{array}$} & $\begin{array}{l}\text { Tramadolo cps SR } 200 \mathrm{mg}+ \\
\text { FANS o paracetamolo* }\end{array}$ & $\begin{array}{l}2 \text { cps SR/die }+ \\
\text { FANS o paracetamolo al bisogno }\end{array}$ & $\begin{array}{l}1,68+ \\
\text { a seconda del } \\
\text { farmaco utilizzato }\end{array}$ & $\begin{array}{l}\text { Range \# 50,4-a } \\
\text { seconda del } \\
\text { farmaco utilizzato }\end{array}$ \\
\hline & $\begin{array}{l}\text { Tramadolo cpr OD } 200 \text { mg + } \\
\text { FANS o paracetamolo* }\end{array}$ & $\begin{array}{l}2 \text { cpr OD } 200 \text { mg da assumere } \\
\text { insieme }+ \\
\text { FANS o paracetamolo al bisogno }\end{array}$ & $\begin{array}{l}1,68+ \\
\text { a seconda del } \\
\text { farmaco utilizzato }\end{array}$ & $\begin{array}{l}\text { Range \# 50,4-a } \\
\text { seconda del } \\
\text { farmaco utilizzato }\end{array}$ \\
\hline
\end{tabular}

\section{Tabella II}

Costo giornaliero di tramadolo ad alte dosi (utilizzate frequentemente nel trattamento del dolore oncologico) secondo il prezzo al pubblico

* impiego di altro farmaco a causa del raggiungimento del massimo dosaggio consentito giornalmente per tramadolo

\# valore minimo: senza episodi di breakthrough pain, valore massimo: 2 episodi di breakthrough pain

età superiore ai 15 anni è affetta da osteoartrosi [23]; la prevalenza di questa patologia supera il $40 \%$ fra i pazienti con età superiore ai 65 anni [24-26]. Soggetti anziani, spesso politrattati costituiscono quindi il paziente "tipo" affetto da questa patologia.

Numerosi studi hanno dimostrato l'efficacia di tramadolo nel trattamento del dolore da moderato a grave associato a osteoartrosi del ginocchio, dell'anca e della colonna vertebrale [27,28]; alcuni trial hanno confrontato diverse formulazioni di tramadolo rilevando che la formulazione OD ha efficacia paragonabile a quella IR, ma il suo utilizzo induce un aumento dell'adesione alla terapia da parte del paziente $[3,29,30]$.

Secondo le linee guida dell'European League Against Rheumatism [31], le principali alternative terapeutiche per il controllo del dolore cronico associato a osteoartrosi sono paracetamolo, FANS e analgesici oppioidi.

Le patologie muscolo-scheletriche correlate al lavoro (work related musculoskeletal disorders: WRMDs) riscontrate più comunemente sono discopatia lombare, lombalgia, sindrome del tunnel carpale, tendiniti della mano, del gomito, della spalla. Tipicamente sono dovute alla presenza di sollecitazioni biomeccaniche ripetute quali vibrazioni, postura incongrua o sforzi eccessivi.

Il $17 \%$ dei lavoratori intervistati durante il Secondo Studio Europeo sulle Condizioni di Lavoro (1996) ha denunciato di avere disturbi muscolo-scheletrici degli arti legati alla loro professione [32]. L'elevata prevalenza e il carattere cronico di queste patologie determinano, oltre a un elevato consumo di risorse dovuto a diagnosi e trattamento, un'imponente spesa indiretta, a carico in parte della società e in parte del cittadino, dovuta essenzialmente alla progressiva limitazione della capacità professionale del paziente e alla perdita di ore di lavoro. Negli Stati Uniti, nel 1989, sono stati spesi circa 11,4 miliardi di dollari per infortuni e malattie professionali riconducibili al gruppo delle patologie muscolo-scheletriche: un terzo della spesa per le cure e gli accertamenti necessari, due terzi per le indennità di assenza dal lavoro [33]. In Germania il costo associato alle malattie del lavoro è stato stimato in circa 28 miliardi di euro, di cui circa 15 miliardi di costi diretti e 13 di costi indiretti. Circa un terzo di quest'ammontare risulta associato a patologie muscolo-scheletriche [34]. In Italia, la reale portata del problema in termini sanitari ed economici non è ancora del tutto chiara, anche se è ragionevole ritenere che non sia troppo dissimile da quella degli altri paesi industrializzati [35]. L'impiego di un efficace trattamento antalgico in grado di migliorare funzionalità fisica, qualità del sonno e qualità di vita in generale, può determinare una riduzione dei costi indiretti conseguenti a tali patologie.

La lombalgia rappresenta uno dei work related disorders più diffusi: essa rappresenta una delle tre cause più comuni di consultazione del medico di medicina generale e una delle 
principali cause di assenteismo dal lavoro $[36,37]$. La maggior parte degli episodi acuti di dolore lombare si risolvono entro tre mesi dalla loro comparsa, tuttavia alcune stime riportano che circa il 5\% della popolazione soffre di lombalgia cronica. In molti di questi casi non viene posta diagnosi eziopatogenica e il trattamento è stabilito su base empirica. I farmaci raccomandati dalle principali linee guida [38$40]$ per il trattamento del dolore cronico associato a lombalgia sono i FANS, gli oppioidi e i miorilassanti. In numerosi studi tramadolo è risultato efficace nel trattamento del dolore associato a lombalgia cronica e nel miglioramento della funzionalità fisica $[30,41,42]$.

Il paziente "tipo" affetto da dolore cronico lombare, così come da lombosciatalgia, sindrome del tunnel carpale o altra patologia correlata al lavoro, è una persona professionalmente e socialmente attiva, in molti casi di età inferiore ai 55 anni [35].

Riportiamo in Tabella III vantaggi e svantaggi legati alle principali opzioni farmacologiche per il trattamento del dolore muscolo-scheletrico cronico.

\section{Dolore neuropatico}

Le neuropatie post-herpetiche e le neuropatie periferiche dovute a diabete rap- presentano le cause più frequenti di dolore neuropatico; esso compare in un'area con deficit sensitivo e può essere bruciante, a fitte $\mathrm{e}$ con sensazione di scottatura, inoltre è accompagnato spesso da dolore non associato a stimoli nocivi (allodinia).

Le stime di prevalenza della neuropatia periferica sono molto variabili: secondo alcuni studi di popolazione, come il UK Multicentre Study e il DiaCAN Multicenter (Germania, Austria e Svizzera), il 17-35\% dei pazienti diabetici soffre di questo disturbo; altre stime riportano valori di prevalenza anche del 54\% [43].

Le sindromi neuropatiche collegate a diabete possono coinvolgere il sistema nervoso periferico e quello autonomo; il tipo più comune è rappresentato dalla Polineuropatia Distale Simmetrica Sensitiva (Distal Symmetric Sensory Polyneuropathy o DSSP) dovuta a una degenerazione assonale distale con una relativa conservazione delle cellule dei gangli delle radici dorsali. Assieme alla vasculopatia periferica, è la causa principale di formazione di ulcere ai piedi e di amputazione delle estremità inferiori [44].

Il trattamento del dolore legato a questa patologia è complesso, soprattutto perché tende ad essere refrattario agli analgesici tradizionali come i FANS e i narcotici [45].

\begin{tabular}{|c|c|c|}
\hline & Vantaggi & Svantaggi \\
\hline Tramadolo & $\begin{array}{l}\text { - sollievo del dolore e miglioramento funzionalità fisica } \\
\text { - elevata compliance per: } \\
\text { semplicità schema posologico e di titolazione } \\
\text { elevata maneggevolezza delle compresse orodispersibili } \\
\text { per controllo breakthrough pain }\end{array}$ & $\begin{array}{l}\text { - tasso di abbandono del } 20 \% \text { per comparsa di effetti } \\
\text { collaterali (nausea/vomito e vertigini) }\end{array}$ \\
\hline $\begin{array}{l}\text { FANS } \\
\text { non selettivi }\end{array}$ & $\begin{array}{l}\text { - azione antinfiammatoria: agisce anche sulle cause del } \\
\text { dolore } \\
\text { - sollievo del dolore }\end{array}$ & $\begin{array}{l}\text { - gastrolesività a lungo termine } \\
\text { - dubbio miglioramento della funzionalità fisica a lungo } \\
\text { termine } \\
\text { - effetto tetto-rischio di tossicità renale } \\
\text { - controindicati in pazienti in terapia anti-ipertensiva o } \\
\text { anticoagulante } \\
\text { - controindicati in pazienti con gastrite o ulcera } \\
\text { - aumento della spesa per associazione con agenti } \\
\text { gastroprotettivi }\end{array}$ \\
\hline FANS & - sollievo del dolore & - possibili reazioni awverse a livello cardio-circolatorio \\
\hline cox-2 & $\begin{array}{l}\text { - azione antinfiammatoria: agisce anche sulle cause del } \\
\text { dolore }\end{array}$ & $\begin{array}{l}\text { - elevati costi } \\
\text { - effetto tetto } \\
\text { - controindicati in pazienti in terapia anti-ipertensiva o } \\
\text { anticoagulante } \\
\text { - controindicati in pazienti con ulcera peptica attiva o } \\
\text { sanguinamento intestinale } \\
\text { - rischio di tossicità renale }\end{array}$ \\
\hline Paracetamolo & $\begin{array}{l}\text { - sollievo del dolore e miglioramento funzionalità fisica } \\
\text { - buona tollerabilità (simile a placebo) fino a 2,6 g/die }\end{array}$ & $\begin{array}{l}\text { - poco adatto a terapia cronica per: } \\
\text { posologia ( } 3 \text { somm/die) } \\
\text { necessità frequenti controlli medici dopo } 10 \mathrm{gg} \text { di terapia } \\
\text { controindicato nei pazienti epatopatici } \\
\text { collocazione in fascia C (a carico del paziente) }\end{array}$ \\
\hline
\end{tabular}




\begin{tabular}{|c|c|c|}
\hline & Vantaggi & Svantaggi \\
\hline $\begin{array}{l}\text { Oppioidi } \\
\text { (tramadolo, } \\
\text { ossicodone) }\end{array}$ & $\begin{array}{l}\text { - miglioramento dell'intensità del dolore e delle funzioni } \\
\text { fisiche e sociali } \\
\text { - miglioramento delle parestesie e dell'allodinia al tatto }\end{array}$ & $\begin{array}{l}\text { - tasso di abbandono della terapia del } 20 \% \text { con } \\
\text { tramadolo per comparsa di effetti collaterali } \\
\text { (nausea/vomito e vertigini) } \\
\text { - limitato uso di ossicodone nel lungo termine per } \\
\text { frequente comparsa di effetti collaterali (costipazione, } \\
\text { nausea, prurito, sonnolenza) }\end{array}$ \\
\hline $\begin{array}{l}\text { Antidepressivi } \\
\text { triciclici } \\
\text { (desipramina, } \\
\text { clomipramina) }\end{array}$ & - sollievo del dolore in una buona percentuale di casi & $\begin{array}{l}\text { - alto tasso di abbandono della terapia per comparsa } \\
\text { eventi awersi (sonnolenza, secchezza delle fauci, } \\
\text { costipazione, aumento di peso) } \\
\text { - necessità di monitorare l'andamento dose/concentr. } \\
\text { per variazioni in base al tipo di metabolizzazione } \\
\text { - controindicati in pazienti con glaucoma, iperplasia } \\
\text { prostatica, ritenzione urinaria, epatopatia, insufficienza } \\
\text { cardiaca, ipotensione ortostatica }\end{array}$ \\
\hline $\begin{array}{l}\text { Anticonvulsivanti } \\
\text { (gabapentin, } \\
\text { lamotrigina, } \\
\text { sodio valproato) }\end{array}$ & $\begin{array}{l}\text { - sollievo del dolore e miglioramento funzionalità fisica } \\
\text { - tasso di abbandono di gabapentin per comparsa di } \\
\text { effetti collaterali simile a placebo }\end{array}$ & $\begin{array}{l}\text { - risultati eterogenei su efficacia di lamotrigina } \\
\text { - efficacia di sodio valproato da confermare nel lungo } \\
\text { termine } \\
\text { - comparsa di sonnolenza e vertigini con gabapentin }\end{array}$ \\
\hline
\end{tabular}

\section{Tabella IV}

Vantaggi e svantaggi legati all'utilizzo di farmaci oppioidi, antidepressivi triciclici e anticonvulsivanti nel trattamento del dolore associato neuropatia diabetica [46]

Uno studio pubblicato recentemente ha revisionato $i$ trial randomizzati e controllati condotti dal 1990 in poi sul trattamento orale del dolore neuropatico in pazienti diabetici [46]. Dall'esame di 24 studi (19 trattamento vs. placebo e 5 comparativi) è emerso che i farmaci in grado di migliorare in modo statisticamente significativo l'intensità del dolore rispetto a placebo sono: desipramina, tramadolo, ossicodone, sodio valproato, gabapentin, acetil-1-carnitina; mexilitina e lamotrigina hanno dato risultati eterogenei nei diversi studi esaminati (probabilmente a causa degli schemi posologici non sempre appropriati).

In base ai dati di efficacia e tollerabilità, gli autori della review hanno svolto alcune considerazioni sulle principali classi di farmaci efficaci nel trattamento del dolore neuropatico; riassumiamo in Tabella IV tali considerazioni.

\section{Dolore oncologico}

Dati di prevalenza forniti dall'OMS indicano che, nel mondo, 17 milioni di persone sono attualmente affette da tumore; di queste, una percentuale che varia fra il $30 \%$, per i pazienti sottoposti a trattamento attivo, e il 70\%, per i pazienti con patologia in fase molto avanzata, è affetta da dolore cronico [2].

La strategia terapeutica proposta dall'OMS per il trattamento del dolore legato a patologie neoplastiche prevede l'utilizzo integrato di diverse procedure e metodiche terapeutiche scelte sulla base della Scala Analgesica a Tre Gradini (Figura 1).

La Scala Analgesica dell'OMS prevede 1'utilizzo di tre categorie di farmaci: non oppioidi, oppioidi per il dolore lieve-moderato e oppioidi per il dolore moderato-severo, con l'integrazione o meno di farmaci adiuvanti e di terapie non farmacologiche in ciascuno dei tre gradini.

L'approccio terapeutico deve essere deciso in base all'intensità del dolore, e non alla sequenzialità dei gradini o alla prognosi del paziente. Inoltre è opportuno che la scelta del farmaco da somministrare dipenda dal tipo di formulazioni disponibili, da considerazioni di farmacocinetica, dalla risposta a precedenti trattamenti e dalla presenza di comorbilità.

L'utilità del II gradino della Scala Analgesica OMS è data dal fatto che circa il

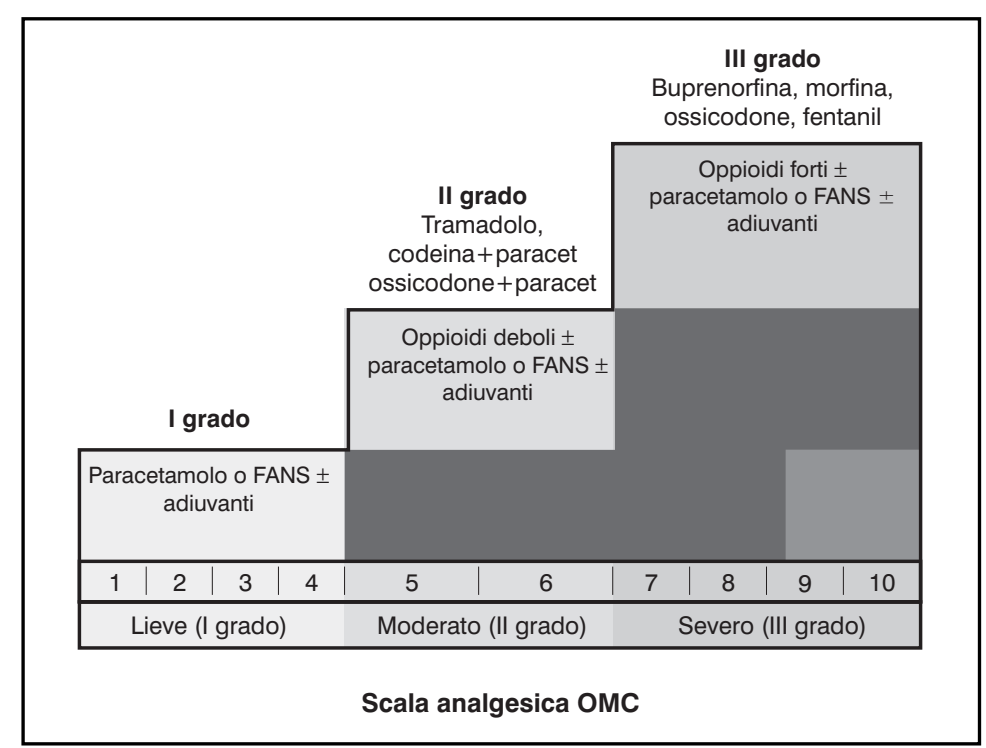

Figura 1

Scala del dolore OMS 
$50 \%$ dei pazienti con dolore cronico oncologico soffre di dolore classificabile come moderato (Figura 2) e che in molti di questi casi la sintomatologia dolorosa non è controllabile con paracetamolo o con FANS.

Inoltre, anche in presenza di dolore lieve (grado I), vi sono numerosi casi in cui l'impiego di anti-infiammatori non steroidei o di paracetamolo è controindicato.

In Tabella $\mathrm{V}$ sono riportati benefici e rischi derivanti dall'utilizzo delle tre opzioni terapeutiche indicate dall'OMS per il trattamento del dolore oncologico moderato (II gradino).

Riassumiamo in Figura 3 gli schemi terapeutici illustrati in precedenza per il trattamento del dolore cronico con tramadolo.

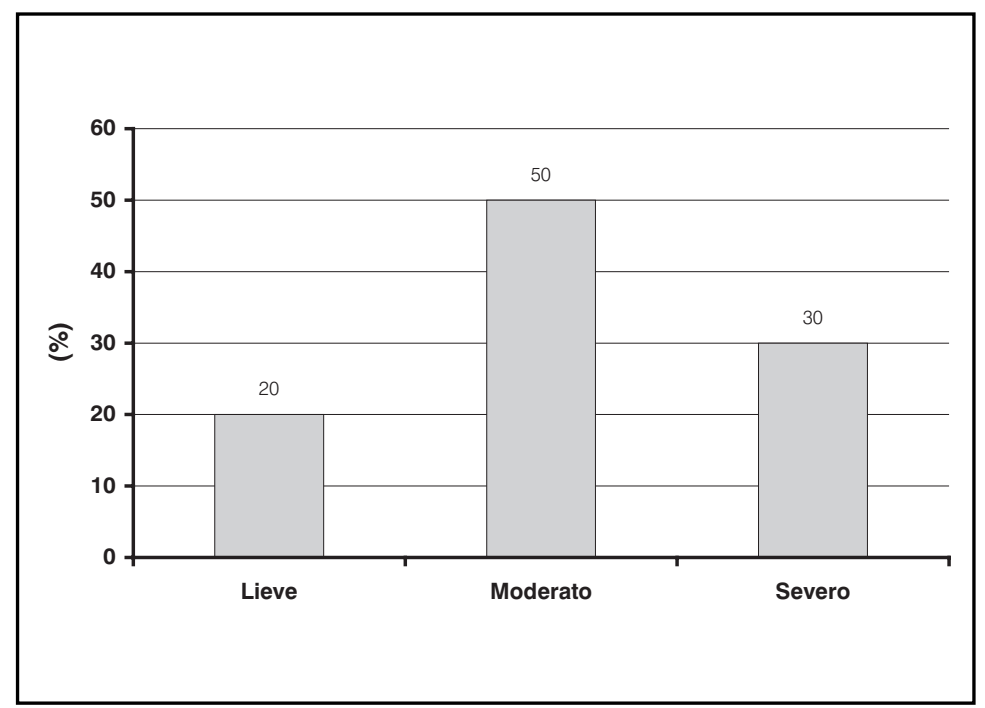

Figura 2

Entità del dolore da cancro [48]

\section{Dolore acuto}

Tramadolo è risultato efficace nel trattamento del dolore acuto associato a traumi, lombalgie, operazioni chirurgiche, infiammazioni e coliche [49-53]. La terapia va cominciata con l'assunzione orale di $50 \mathrm{mg}$ di tramadolo IR; se dopo 30-40 minuti il dolore persiste con uguale intensità si può assumere un'altra dose da $50 \mathrm{mg}$. Se è necessario continuare il trattamento, la posologia raccomandata è di 1 o 2 dosi da 50 mg ogni 4-6 ore, senza superare il dosaggio giornaliero di $400 \mathrm{mg}$; la durata della terapia deve essere stabilita in funzione della necessità terapeutica. Per quanto riguarda le varie formulazioni orali di tramadolo, il dolore acuto può essere gestito mediante l'utilizzo delle capsule tradizionali, delle gocce e delle compresse orodispersibili. Quest'ultima formulazione, grazie alle sua maneggevolezza può comportare, come detto in precedenza, vantaggi per quanto riguarda praticità e sicurezza di somministrazione, a fronte di una spesa giornaliera uguale a quella sostenuta utilizzando le altre formulazioni di tramadolo IR (Tabella VI).

\section{CONCLUSIONI}

Attualmente si stima che in Italia circa 10 milioni di persone soffrano di un dolore cronico benigno, mentre circa un milione di pazienti siano affetti da dolore cronico oncologico. Se a questi dati si somma il numero di persone che giornalmente subiscono un intervento chirurgico o un trauma, che soffrono di colica o di lombalgia, ci si può rendere conto di come il dolore rappresenti un problema clinico, economico e sociale di ampia portata [2].

Oltre ai costi diretti legati alla gestione del dolore, rappresentati soprattutto dal costo

\begin{tabular}{|c|c|c|}
\hline & Vantaggi & Svantaggi \\
\hline Tramadolo & $\begin{array}{l}\text { - sollievo del dolore } \\
\text { - basso potenziale di abuso, dipendenza e tolleranza } \\
\text { - elevata compliance per: } \\
\text { semplicità schema posologico e di titolazione } \\
\text { (monosomministrazione/die) } \\
\text { elevata maneggevolezza delle compresse orodispersibili } \\
\text { per il controllo del dolore incidente }\end{array}$ & $\begin{array}{l}\text { - tasso di abbandono della terapia del } 20 \% \text { per } \\
\text { comparsa effetti collaterali (nausea/vomito e vertigini) }\end{array}$ \\
\hline $\begin{array}{l}\text { Associazioni } \\
\text { Oppioide debole/ } \\
\text { paracetamolo }\end{array}$ & $\begin{array}{l}\text { - sollievo del dolore } \\
\text { - associazioni con effetto analgesico maggiore e più } \\
\text { duraturo rispetto ai singoli componenti }\end{array}$ & $\begin{array}{l}\text { - posologia che richiede tre/quattro somministrazioni al } \\
\text { giorno } \\
\text { - controindicate nei pazienti epatopatici e in pazienti con } \\
\text { insufficienza respiratoria } \\
\text { - rischio di sviluppo di tolleranza e di dipendenza } \\
\text { - comparsa di effetti collaterali (stipsi, sonnolenza) } \\
\text { - bassa maneggevolezza di codeina/paracetamolo } \\
\text { - comparsa di sindrome da astinenza in caso di } \\
\text { interruzione dopo trattamento prolungato con ossicodone }\end{array}$ \\
\hline
\end{tabular}

\section{Tabella V}

Vantaggi e svantaggi legati all'utilizzo di tramadolo e delle associazioni di oppioidi deboli (codeina e ossicodone) e paracetamolo nel trattamento del dolore oncologico moderato 


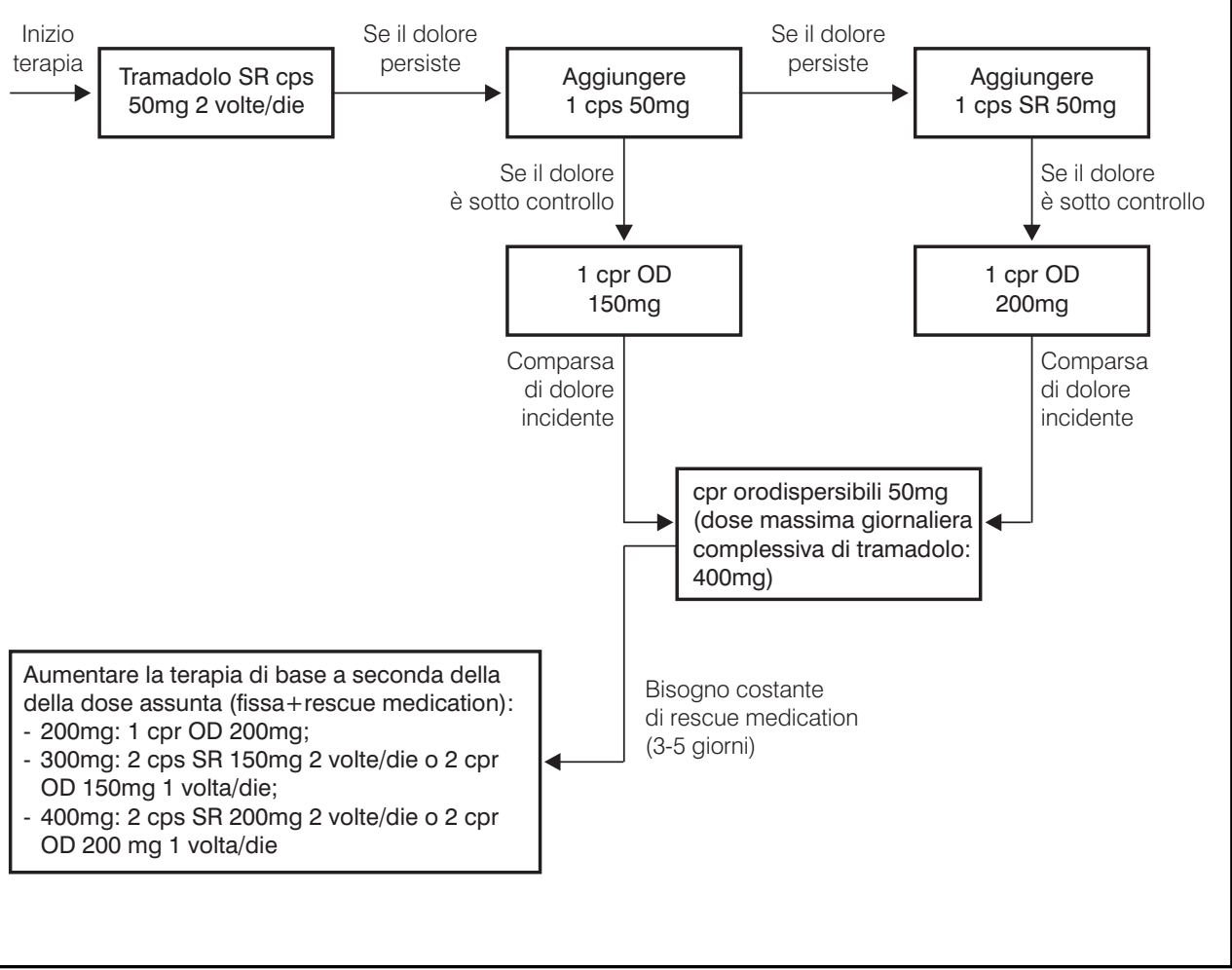

della terapia antidolorifica e dei farmaci adiuvanti, dal costo di gestione degli effetti collaterali di tali farmaci e dalle visite mediche e specialistiche, nei paesi industrializzati il dolore cronico è riconosciuto come una delle principali cause d'inabilità professionale e quindi di costo sociale [35].

Alcuni ricercatori americani hanno stimato mediante il database del National Health Interview Survey (NHIS) le patologie più frequentemente associate ad assenteismo sul lavoro e ridotta capacità produttiva (Figura 4) [33].

Come si evince dalla Figura 4, patologie muscolo-scheletriche croniche associate a sintomatologia dolorosa come la lombalgia cronica e l'osteoartrosi rappresentano globalmente la causa primaria di quasi il $30 \%$ dei casi di inabilità professionale.

La perdita di giorni lavorativi, così come la riduzione della produttività o della qualità del lavoro rappresentano costi indirettamente legati al sintomo dolore, i quali gravano in parte sul nucleo familiare del paziente e in parte sulla collettività. Molto spesso a tali costi occorre sommare quelli relativi all'aiuto e all'assistenza che una persona con dolore cronico necessita per portare a termine normali mansioni quotidiane, come la cura della casa o dei figli.

Nell'analisi dei costi associati alla "patologia dolore", un ruolo predominante è rivestito dai costi cosiddetti intangibili; essi sono difficilmente valorizzabili e sono rappresentati dall'impatto che una condizione patologica, o il

\section{Tabella VI} compresse orodispersibili suo trattamento, ha sulla qualità di vita del paziente.

Il dolore, soprattutto quello cronico, intacca infatti lo svolgimento delle attività quotidiane, sociali e familiari, aumenta nervosismo e stress e diminuisce la qualità del sonno con ulteriore peggioramento del quadro clinico e psicologico.

I benefici clinici derivanti da una terapia analgesica efficace hanno dunque ricadute positive sulla qualità di vita e sui costi indiretti associati alla patologia. Essa infatti può migliorare la funzionalità fisica del paziente, riducendo i giorni di lavoro persi, la sua partecipa-

Figura 3

Schemi terapeutici consigliati per il trattamento del dolore cronico con tramadolo

\begin{tabular}{|c|c|c|c|}
\hline $\begin{array}{l}\text { Dose } \\
\text { giornaliera }\end{array}$ & $\begin{array}{c}\text { Forma } \\
\text { farmaceutica }\end{array}$ & $\begin{array}{l}\text { Schema } \\
\text { posologico }\end{array}$ & $\begin{array}{c}\text { Costo } \\
\text { giornaliero (euro) }\end{array}$ \\
\hline \multirow[t]{3}{*}{$200 \mathrm{mg}$} & $\begin{array}{l}\text { Tramadolo } 20 \mathrm{cpr} \\
\text { orodispersibili } 50 \mathrm{mg}\end{array}$ & $4 \mathrm{cpr} / \mathrm{die}$ & 0,92 \\
\hline & $\begin{array}{l}\text { Tramadolo gtt } 10 \mathrm{ml} \\
100 \mathrm{mg} / \mathrm{ml}\end{array}$ & $\begin{array}{c}20 \mathrm{gtt}(50 \mathrm{mg}) \\
4 \text { volte/die }\end{array}$ & 0,92 \\
\hline & $\begin{array}{l}\text { Tramadolo } 20 \mathrm{cps} \\
50 \mathrm{mg}\end{array}$ & 4 cps/die & 0,92 \\
\hline \multirow[t]{3}{*}{$400 \mathrm{mg}$} & $\begin{array}{l}\text { Tramadolo } 20 \mathrm{cpr} \\
\text { orodispersibili } 50 \mathrm{mg}\end{array}$ & $8 \mathrm{cpr} / \mathrm{die}$ & 1,84 \\
\hline & $\begin{array}{l}\text { Tramadolo gtt } 10 \mathrm{ml} \\
100 \mathrm{mg} / \mathrm{ml}\end{array}$ & $\begin{array}{l}20 \mathrm{gtt} 8 \text { volte/die } \\
(=4 \mathrm{ml})\end{array}$ & 1,84 \\
\hline & $\begin{array}{l}\text { Tramadolo } 20 \mathrm{cps} \\
50 \mathrm{mg}\end{array}$ & $8 \mathrm{cps} / \mathrm{die}$ & 1,84 \\
\hline
\end{tabular}

Costo giornaliero del trattamento del dolore acuto con tramadolo in gocce o in 


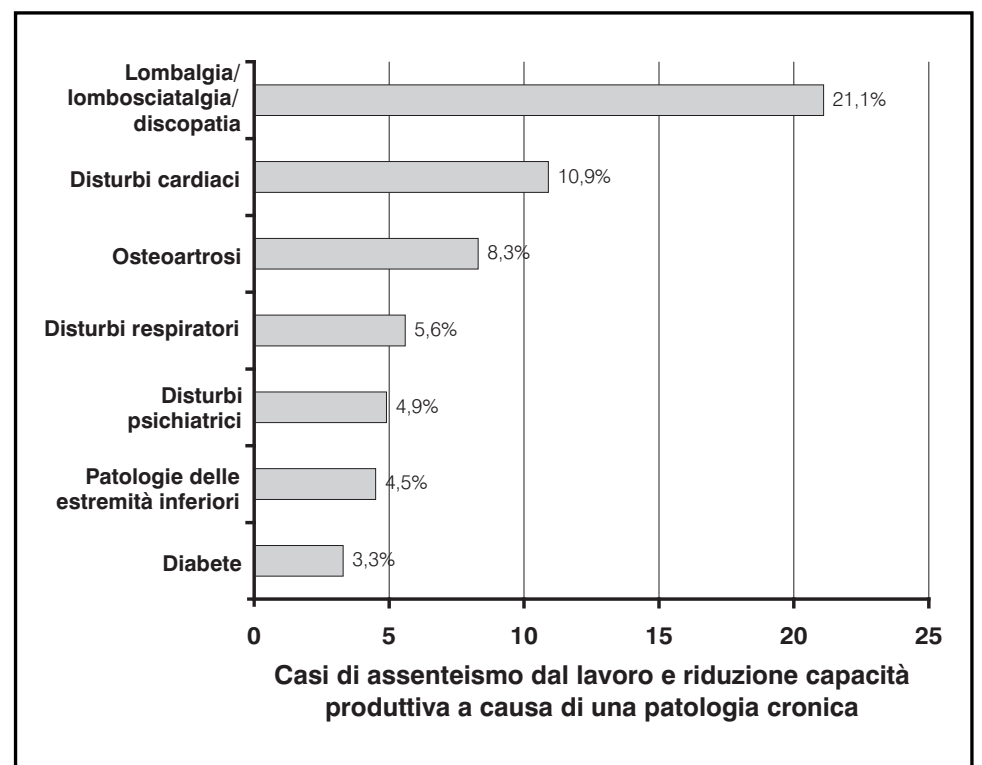

Figura 4

Condizioni croniche che rappresentano le più frequenti cause di inabilità professionale (modificata da [33])

zione alla vita sociale e la sua indipendenza. Alcuni pazienti, ad esempio, possono avere bisogno dell'aiuto di un care-giver (familiari o personale qualificato), e l'effetto positivo sul- la qualità di vita del paziente si riflette, necessariamente, anche su quella degli assistenti e, in termini più ampi, della collettività.

Un paziente senza dolore, inoltre, collabora maggiormente con il personale medico e la rapida ripresa della funzionalità fisica, riduce anche tutti i rischi legati all'immobilità e le conseguenze psicologiche.

Oltre a essere intrinsecamente efficace, per massimizzare l'efficienza prescrittiva occorre che l'aderenza alla terapia sia elevata. La scelta di una determinata forma farmaceutica, influenzando lo schema posologico e le modalità d'assunzione, può migliorare la compliance e la soddisfazione del paziente; ciò permette il raggiungimento dell'obiettivo terapeutico che, a sua volta, contribuisce ulteriormente ad aumentare la compliance. Le nuove formulazioni di tramadolo in compresse orodispersibili e once-daily hanno contribuito a migliorare l'efficienza della terapia analgesica, permettendo di personalizzare gli schemi terapeutici e incontrare maggiormente le esigenze delle diverse tipologie di pazienti.

Questo si riflette in un miglioramento della qualità di vita del paziente e del suo nucleo familiare e in una conseguente riduzione di costi indiretti e intangibili.

\section{BIBLIOGRAFIA}

1. www.ministerosalute.it/dettaglio/pdPrimoPiano.jsp?id=44\&sub=6\&lang=it

2. www.ministerosalute.it/resources/static/primopiano/sollievo/opuscolo.doc

3. Bodalia B. A comparison of the pharmacokinetics, clinical efficacy, and tolerability of once-daily tramadol tablets with normal release tramadol capsules. J Pain Symptom Manage. 2003 Feb;25(2):142-9.

4. Tagarro I, Vauzelle-Kervroedan F, Dìez MC. Pharmacokinetic assessment of a fast-release orodispersible tramadol tablet compared to a conventional tramadol capsule. Arzneim. Forsch./Drug Res. 54, No. 5. 293-297 (2004).

5. Cnota PJ et al. Formulazioni di tramadolo SR. Confronto dei parametri farmacocinetici: dose ad unità multiple (capsula) vs dose a unità singola (compressa). Clin Drug Invest 2005; 25(7); 435-443.

6. Lintz W et al. Bioavailability of enteral tramadol formulations. 1st communication: capsules. Arzneimittelforschung. 1986 Aug;36(8):1278-83.

7. Raber $\mathrm{M}$ et al. Pharmacokinetic properties of tramadol sustained-release capsules. 2nd communication: investigation of relative bioavailability and food interaction. Arzneimittelforschung. 1999 Jul;49(7):588-93.

8. Raber $\mathrm{M}$ et al. Analgesic efficacy and tolerability of tramadol $100 \mathrm{mg}$ sustained-release capsules in patients with moderate to severe chronic low back pain. Clin Drug Invest 1999; Jun; 17(6): 415-23.

9. Tiwari SB et al. Controlled release formulation of tramadol hydrochloride using hydrophilic and hydrophobic matrix system AAPS. Pharm Sci Tech 2003. 4(3). 
10. ADAMON $^{\circledR}$ - Riassunto caratteristiche del prodotto.

11. Richter A et al. The impact of reducing dose frequency on health outcomes. Clin Ther. 2003 Aug;25(8):2307-35; discussion 2306.

12. Claxton AJ et al. A systematic review of the associations between dose regimens and medication compliance. Clin Ther. 2001 Aug;23(8):1296-310.

13. Mattia C et al. Il tramadolo per il trattamento del dolore post-operatorio in chirurgia ortopedica maggiore. Min. Anest. 1997 Vol. 63 Suppl. 1.

14. Tagarro et al. Effetti sulla tollerabilità di tramadolo somministrato secondo un semplice schema terapeutico che prevede un aumento posologico. Clin Drug Invest 2005; 25(1);23-31.

15. Assessment and management of pain. Registered Nurses Association of Ontario - Professional Association. 2002 Nov. 142 pages.

16. Control of pain in patients with cancer. A national clinical guideline. Scottish Intercollegiate Guidelines Network - National Government Agency [Non-U.S.]. 2000 Jun. 61 pages.

17. Tradonal ${ }^{\circledR-R i a s s u n t o ~ C a r a t t e r i s t i c h e ~ d e l ~ P r o d o t t o . ~}$

18. Mercadante S et al. Episodic (breakthrough pain) Consensus conference of an expert working group of the European Association for Palliative Care. Cancer 2001.

19. Mannoni A et al. Epidemiological profile of symptomatic osteoarthritis in older adults: a population based study in Dicomano, Italy. Ann Rheum Dis 2003; 62: 576-8.

20. Corti MC, Rigon C. Epidemiology of osteoarthritis: prevalence, risk factors and functional impact. Aging Clin Exp Res 2003; 15: 359-63.

21. Mantyselka P et al. Pain as a reason to visit the doctor; a study in Finnish primary health care. Pain 2001; 89: $175-80$.

22. Salaffi F et al. Analysis of disability in knee osteoarthritis. Relationship with age and psychological variables but not with radiographic score. I J Rheumatol 1991; 18: 1581-6.

23. US National Health Interview Survey. National Center for Health Statistic, 2002.

24. Catala E et al. Prevalence of pain in the Spanish population: telephone survey in 5000 homes. Eur J Pain. 2002;6(2):133-40.

25. Gagliese L, Melzack R. Chronic pain in elderly people. Pain. 1997 Mar;70(1):3-14.

26. Damian J et al. Health and functional status among elderly individuals living in nursing homes in Madrid. Gac Sanit. 2004 Jul-Aug;18(4):268-74.

27. Bijlsma JW. Analgesia and the patient with osteoarthritis. Am J Ther. 2002 May-Jun;9(3):189-97.

28. Pavelka K. Treatment of pain in osteoarthritis. Eur J Pain. 2000; 4 Suppl A:23-30

29. Adler et al. A comparison of once-daily tramadol with normal release tramadol in the treatment of pain in osteoarthritis. J Rheumatol. 2002 Oct;29(10):2196-9.

30. Casals M, Samper D. Efectividad, tolerabilidad y calidad de vida en el tratamiento del dolor cronico no oncologico, con tramadol de liberacion controlada en dosis unica diaria. Rev. Soc. Esp. Dolor 11: 129-140, 2004.

31. Punzi L et al. Italian consensus on Eular 2003 recommendations for the treatment of knee osteoarthritis. Reumatismo. 2004 Jul-Sep;56(3):190-201.

32. http://europa.eu.int/comm/public_opinion/archives/ebs/ebs_096_en.pdf

33. Stoddard, S et al. Chartbook on Work and Disability in the United States. 1998. An InfoUse Report. Washington, D.C.: U.S. National Institute on Disability and Rehabilitation Research.

34. Boedeker W et al. The costs of work related diseases in germany 1998 (http://www.arbejdsmiljo.dk/wien/billeder/ tyskerne\%2028\%20mia\%20euro.pdf).

35. Mattioli S et al. Il gruppo collaborativo MODS: la sindrome del tunnel carpale. Atti del VII Congresso nazionale della Società Italiana di Ergonomia, Firenze, 26-28 settembre 2001, pp. 579-586.

36. Atkinson JH, Chronic Back Pain: Searching for Causes and Cures. 2004 Volume 31.no:12 The Journal of Rheumatology.

37. Jermyn RT. A nonsurgical approach to low back pain. JAOA. Vol 101, no 4 Supplement to April 2001.

38. Cole A, Herring S. The low back pain handbook: a practical guide for the primariy care clinician. Philadelphia, Pa: Handley \& Belfus, Inc; 1997; pp 31, 39, 59-90, 92. 
39. Adult low back pain. Bloomington (MN): Institute for Clinical Systems Improvement (ICSI); 2004 Sep. 63 pages.

40. Unremitting low back pain. In: North American Spine Society phase III clinical guidelines for multidisciplinary spine care specialists. North American Spine Society - Medical Specialty Society. 2000. 96 pages.

41. Muller FO et al. Comparison of the efficacy and tolerability of a paracetamol/codeine fixed-dose combination with tramadol in patients with refractory chronic back pain. Arzneimittelforschung. 1998 Jun;48(6):675-9.

42. Schnitzer TJ et al. Efficacy of tramadol in treatment of chronic low back pain. J Rheumatol. 2000 Mar;27(3):772-8.

43. Ziegler, D. Diagnosis and management of diabetic peripheral neuropathy. Diabetic Medicine. 1996; 13:S34-38.

44. Thomas, P. K. Diabetic neuropathy: mechanisms and future treatment options. Journal of Neurology, Neurosurgery \& Psychiatry 1999; 67:277-281.

45. Rawn T et al. Gabapentin or amitriptyline for painful diabetic neuropathy? Canadian Family Physician. 2000; 46:2215-2217.

46. Adriaensen $\mathrm{H}$ et al. Critical review of oral drug treatments for diabetic neuropathic pain-clinical outcomes based on efficacy and safety data from placebo-controlled and direct comparative studies. Diabetes Metab Res Rev. 2005 May-Jun;21(3):231-40.

47. ahttp://www.ministerosalute.it/resources/static/primopiano/257/AIFAOppiacei.pdf

48. https://www.apss.tn.it/Allegati/DOC_290167_0.pdf

49. Pendeville PE et al. Double-blind randomized study of tramadol vs. paracetamol in analgesia after day-case tonsillectomy in children. Eur J Anaesthesiol. 2000 Sep;17(9):576-82.

50. Moore RA, McQuay. Single-patient data meta-analysis of 3453 postoperative patients: oral tramadol versus placebo, codeine and combination analgesics. Pain. 1997 Feb;69(3):287-94.

51. McQuay H, Edwards J. Meta-analysis of single dose oral tramadol plus acetaminophen in acute postoperative pain. Eur J Anaesthesiol Suppl. 2003;28:19-22.

52. Lehmann KA. Tramadol in acute pain Drugs. 1997;53 Suppl 2:25-33.

53. Radbruch L, Grond S, Lehmann KA. A risk-benefit assessment of tramadol in the management of pain. Drug Saf. 1996 Jul;15(1):8-29. 